\title{
Dos
}

\section{Seis factores \\ tecnológicos}

DOI: 10.29236/sistemas.n158a7

\section{En la educación del futuro.}

\section{Resumen}

En un momento histórico cuando el uso de las tecnologías educativas conlleva a la innovación pedagógica y a la creación de nuevas estrategias didácticas para la enseñanza y aprendizaje dentro de las instituciones educativas, es fundamental poder conocer cómo la Infraestructura, Entorno, Cohesión, Conectividad, Innovación y Analítica serán los seis factores imprescindibles cuando de educación futura hablamos.

A continuación, abordaremos distintas miradas basadas en las realidades y el día a día de las diferentes empresas con propósito de formación y conoceremos cómo deberíamos prepararnos para responder a las necesidades educativas y así proyectar los intereses de formación durante los próximos 10 años.

Dentro del presente artículo usted tendrá la posibilidad de analizar una propuesta de incorporación de tecnologías, con el fin de sostener una educación virtual a futuro, con calidad y de fácil adopción por parte de nuestros estudiantes y profesores, reduciendo los índices de deserción estudiantil y mejorando sustancialmente la experiencia del usuario en su entorno de aprendizaje.

Es una invitación a reflexionar y repensar sobre cómo dentro de su institución se están abordando estos seis factores con el fin de lograr el punto de equilibrio entre tecnología, educación y usuarios en procesos de formación con apoyo de TI, para lograr ser competentes e innovadores en la educación del futuro. 
Educación, Futuro, Virtual, Plataformas, innovación

\section{Manuel Antonio Meléndez Andrade}

\section{Introducción}

En un mundo cambiante donde nuestra situación actual de pandemia y las distintas modalidades de educación reinan, es difícil poder comprender el sistema educativo como un todo, por eso me he puesto en la tarea de desarrollar y presentarles seis factores tecnológicos esenciales en la educación del futuro.

Cuando me solicitaron escribir este artículo para hablar sobre la educación del futuro, sentí estar frente a un gran reto, pues como ingeniero de sistemas y telecomunicaciones, es sumamente complejo pretender hablar de educación futura debido a lo amplio del tema, por esta razón inicié una búsqueda sobre cuál sería una temática interesante para discutir.

Durante este texto podremos aprender a prepararnos para proyectar la educación del futuro a diez años, basándonos en seis factores tecnológicos que brindarán una perspectiva clara y muy holística de la educación en un futuro no muy lejano.

Este escrito pretende proponer un sistema capaz de crear una sinergia entre las partes y lograr lo que toda empresa o institución desea: una educación con calidad.
La ilustración 1 muestra en orden prioritario y secuencial cómo se deben abordar los factores para lograr el equilibrio entre lo tecnológico y el propósito educativo.

El primer factor INFRAESTRUCTURA, planteará la importancia de las plataformas virtuales en el mundo educativo, cómo éstas deben de evolucionar y adaptarse a los cambios que nuestra realidad demanda en las instituciones con propósitos formativos.

El segundo factor ENTORNO, brindará las ideas necesarias para implementar una plataforma virtual, orientada a la experiencia del aprendizaje, priorizando la experiencia del usuario, los contenidos adaptativos, flexibilidad y apertura a futuras integraciones.

Desde el tercer factor COHESIÓN, se pretende entender cómo las distintas áreas de una institución deben estar armonizadas para lograr procesos con calidad cuando de tecnologías educativas hablamos.

En el cuarto factor CONECTIVIDAD, trataremos de explicar cómo se deben incorporar nuevas competencias digitales, basadas en el uso de las nuevas herramientas de video conferencia, con la intención de lograr una comunicación eficien- 


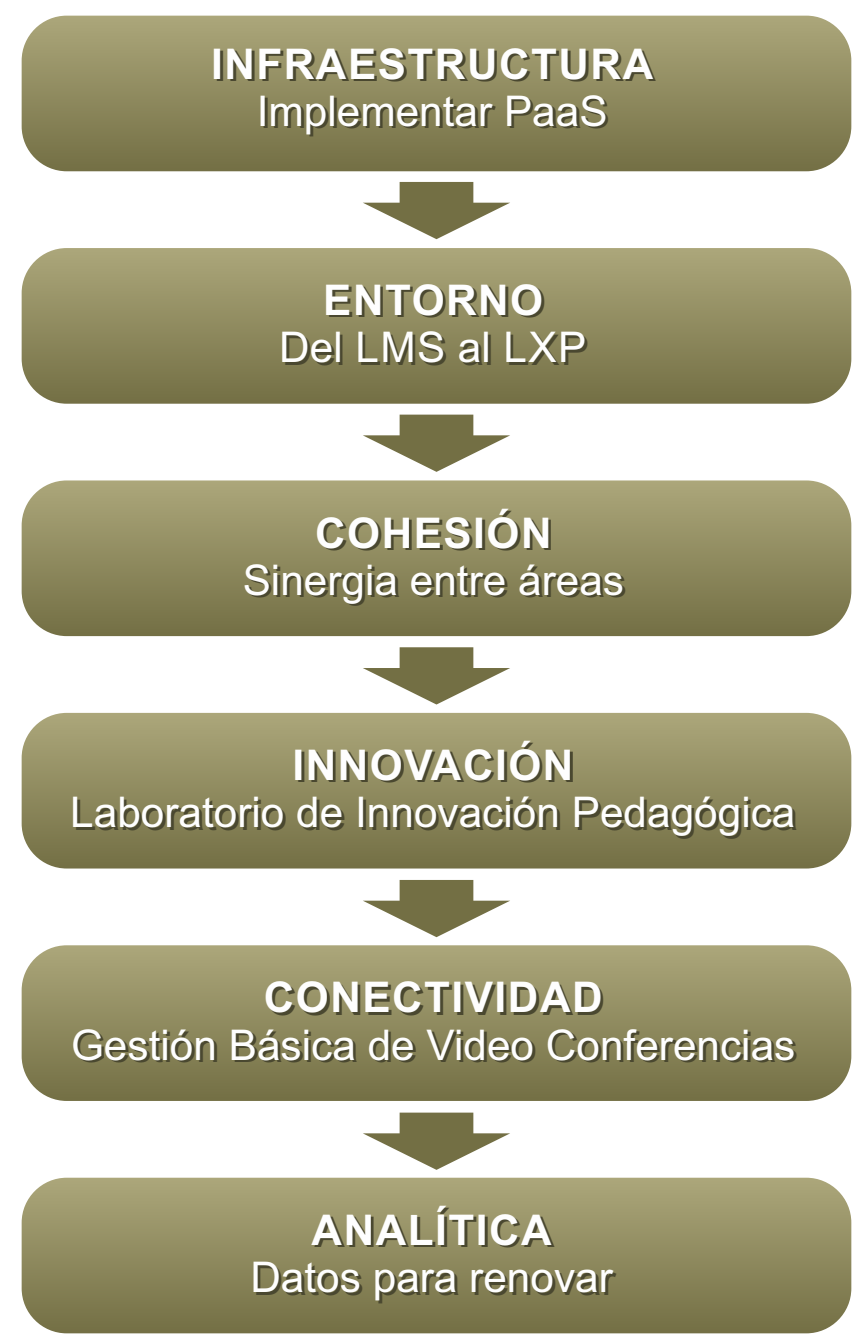

Ilustración 1. Propuesta distribución de los seis factores tecnológicos esenciales en la educación del futuro.

te y eficaz entre las partes, y así asegurar que las estrategias de impartición de contenidos asincrónicas o remotas sean un éxito.

Nuestro quinto factor INNOVACIÓN, está orientado a la creación de un Laboratorio de Innovación Pedagógica, con el fin de implementar Testing Educativo en las empresas con propósito de formación, y así, lograr posicionarse como pioneros en la creación y publicación de prácticas y modelos innovadores para una educación a futuro.

Finalmente, el sexto factor ANALÍTICA, ayudará a entender cómo gran cantidad de datos existentes 
en las diferentes aplicaciones software, o plataformas educativas nos ayudan no solo a tomar decisiones, sino a prepararnos para la renovación de los programas bajo los principios de calidad, sostenibilidad y pertinencia.

Una vez profundicemos en los seis factores esenciales, se espera que usted, señor lector, logre entender este macro sistema de incorporación de tecnologías educativas y que su empresa o situación logre estar preparada para los retos de una educación con calidad en los próximos 10 años.

\section{Infraestructura: PaaS la tendencia en el futuro de la educación}

Siempre que nos referimos a la educación virtual y al futuro de la educación, pensamos en cuál será y si nuestra plataforma tendrá la capacidad de soportar el modelo; para ello, existen diferentes estándares que la mayoría de las instituciones implementan, entre ellos:

InHouse: Modelo donde la institución soporta la infraestructura y el despliegue del servicio, siendo éste, el más autónomo, pero genera un alto costo dado que se debe de velar por la disponibilidad del servicio e invertir en prácticas y procedimientos para brindar un servicio con calidad.

Saas: Los Software As A Services permiten como su nombre lo indica, tener a un proveedor o tercero ca- paz de asegurar la infraestructura necesaria para garantizar un buen servicio, permitiendo a la institución realizar actividades front-end, descargando las tareas de back-end al proveedor. Esta práctica es una de las más comunes; sin embargo, genera poca libertad para realizar procedimientos y mejoras, toda vez que limitan las garantías del servicio brindadas por el proveedor.

Entendiendo estos modelos, podemos comprender que el futuro de la educación está en los PaaS (Platform As A Service), siendo el factor (INFRAESTRUCTURA) el primero en la lista del desarrollo de este artículo. Las PaaS son un modelo que contiene los servicios, la infraestructura y el soporte respectivo en la nube. Mediante las Paas, nuestra preocupación de caídas debería desaparecer y los pedagogos y académicos podrán enfocarse en la innovación, dejando a un lado las preocupaciones relacionadas con el crecimiento y disposición del servicio de la plataforma o limitando su quehacer por pensar en la estabilidad del servicio.

Las PaaS nacen de la experiencia y la robustez de empresas como Amazon con su servicio AWS, en las que su core es la infraestructura en la nube pero, que a su vez y debido a las nuevas tendencias educativas, a situaciones de pandemia y a la alta demanda, se han logrado especializar también, en el correcto y adecuado despliegue de un entorno necesario para lograr que la 
educación en un futuro sea accesible, liviana y robusta, capaz de soportar miles de usuarios concurrentes e internacionalizando el aprendizaje; estas plataformas serán capaces de ser desplegadas con las mejoras prácticas, para la mejor disposición de los contenidos en cualquier lugar, sin limitantes, desde que se tenga una conexión de internet estable.

\section{Entorno: del LMS al LXP}

Si bien es cierto que hoy las Ilamadas "plataformas virtuales" son y han sido el entorno necesario para soportar y gestionar los objetos y contenidos virtuales para el aprendizaje, reflejados en los cursos virtuales y nuestras clases en modalidad remota, mixta o de apoyo a la presencialidad, es necesario realizar una actualización a los propósitos de estas y adoptar una flexibilidad en la forma que los contenidos son mostrados a nuestros estudiantes. Es preciso tener avances en la experiencia del usuario frente al desarrollo del aprendizaje, por esto debemos de hablar del LXP.

Los LXP de su sigla (Learning Experience Platform) son aquellas plataformas orientadas a la experiencia en el desarrollo del aprendizaje; es decir, son plataformas donde prima el usuario final (los estudiantes) y la forma en que se presenta dicho contenido está sujeta al desarrollo progresivo del aprendizaje y a los gustos e intereses del estudiante en su entorno virtual o remoto de trabajo.
Hablar de la transición de los LMS a los LXP como apoyo al factor 2 (ENTORNO), conlleva a entender que no podemos hablar de la educación del futuro, si no tenemos una plataforma preparada para los retos que nos trae la educación en modalidad virtual, remota, blended y presencial con apoyo de virtualidad.

Así mismo, es necesario dentro de las instituciones, realizar un cambio en la forma en que se comprenden las plataformas virtuales, dado que hoy en día, las unidades o departamentos de educación virtual son ejes centrales capaces de aportar a las tendencias del futuro de la educación.

Al lograr la transición de los LMS a los LXP en una institución o empresa, se podrán obtener algunos de los beneficios que muy seguramente permitirán a las diferentes áreas ubicarse en ese punto de quiebre ideal, en el que la innovación, los medios, el entorno de trabajo y la forma en que se dispone la información, dejarán de ser una preocupación para convertirse en un punto de éxito y disrupción educativa hacia la adquisición de estrategia didácticas innovadoras.

Algunos de los beneficios de los LXP son:

- UX/UI user: Al ser plataformas orientadas a los usuarios, permitirán que todos puedan interactuar facialmente y a su vez extraer los 
contenidos de manera más natural; es decir, mayor consumo de información por parte de los usuarios profesor, estudiante y administradores, menor cantidad de horas invertidas en capacitaciones y talleres para el correcto uso y apropiación.

- Aprendizaje colaborativo: Las LXP se caracterizan por ser facilitadores del aprendizaje colaborativo, pues uno de los ejes de su núcleo es fomentar los trabajos asociativos, fomentar la participación, la democracia en el aula virtual y las interacciones entre sus usuarios.

- Analítica en tiempo real: Como objeto esencial de los LXP es el fácil acceso y consulta de los datos y registros de las distintas acciones por parte de los usuarios, así como generar predicciones y reducir la brecha de la deserción a través de la toma de decisiones anticipadas relacionadas con el quehacer de nuestros estudiantes.

- Contenidos adaptativos: Muy de la mano de las estadísticas, tendremos la posibilidad de generar contenidos adaptativos, los cuales se generarán y se mostrarán a nuestros estudiantes a la par del ritmo en que el usuario desarrolla las unidades temáticas y el nivel de dificultad de estas, es decir, el estudiante verá de forma medida los contenidos de acuerdo con su destreza frente a la realización de las actividades y contenidos dispuestos en estas.
- Flexibilidad en las diferentes integraciones: Una de las novedades de las LXP es la posibilidad de generar fácilmente integraciones con diferentes aplicaciones, plugins o complementos, entre los cuales se destaca el no uso de actividades SCORM y mejoras en las integraciones LTI para una mayor facilidad de cara a las estrategias que requiera la institución o empresa.

\section{Cohesion: Sinergia educativa entre las áreas}

Uno de los factores que casi siempre se pasa por alto en el momento de hablar de la Educación del Futuro, está relacionado con esos límites, contextos y la cohesión entre las diferentes áreas.

Siempre deberá existir una sinergia entre las distintas áreas, departamentos y unidades dentro de una organización, cuando de educación virtual del futuro se habla, pues es así como realmente los esfuerzos mancomunados podrán dar el efecto deseado en la educación.

Un ejemplo claro de la sinergia entre áreas que debe de existir, se puede entender en el siguiente caso:

Un estudiante fue aceptado por la institución de forma tardía por el programa y desde el primer momento de su aceptación, se desprenden situaciones como las siguientes en las que él requiere:

- Tener activo sus datos de acceso. (áreas de Registro y Control) 
- Acceso a su plataforma virtual. (Sistemas de sincronización como UXXI, Banner u otros)

- Capacitación en el uso de plataformas virtuales (Centros e-elearning)

- Conocer los canales de soporte (Centro de atención al usuario)

- Ponerse al día en las actividades académicas (Empalme con el profesor)

Situaciones como estas, la cuales son normales en el día a día, conllevan a que tanto el estudiante como las diferentes áreas entren a trabajar inmediatamente en paralelo para asegurar que solo ese estudiante tenga su estado ideal para iniciar un proceso de aprendizaje desde las plataformas virtuales.

En la educación del futuro, en el momento de hablar de sinergia académica entre las áreas, se deberá siempre tener presente el factor (COHESION), y en sus unidades crear o implementar una unidad transversal, capaz de direccionar las implementaciones tecnológicas educativas y crear el balance necesario para que las unidades se entiendan, y a su vez establecer los lineamientos y buenas políticas de la educación virtual.

Un caso de éxito en el país es la Dirección de Educación Digital (DED) de la Universidad del Rosario, en donde a través de los años se ha logrado articular las diferentes áreas para preparar a la institución en el proceso de adopción tecnológica e innovación educativa; trabajando de la mano y al mismo nivel de la Dirección Académica, la Dirección de estudiantes, el Área de Tecnología, Registro y las Coordinaciones de los diversos programas y escuelas, en procura de un mismo objetivo, una institución capaz de brindar calidad en el proceso de enseñanza aprendizaje, apoyados en el buen uso de las tecnologías educativas y modelos de virtualización claros para una educación del futuro con responsabilidad.

\section{Gbv - gestión básica de videoconferencias - conectividad}

En días donde la educación virtual ha sido nuestro único medio para lograr dar continuidad a los procesos de formación, es esencial buscar que las competencias digitales básicas en el uso de tecnologías para la educación, se incorporen fácilmente y que al final logren su cometido en el aprendizaje en nuestros estudiantes. Por eso es básico que todos los usuarios de tecnologías educativas tengan las competencias mínimas desde el primer acercamiento a las instituciones. Es así como el factor (CONECTIVIDAD) es esencial al pensar sobre la educación del futuro, de la mano con la gestión básica de videoconferencias.

Las habilidades y competencias en el uso de estas herramientas permitirán una mayor libertad para impartir los contenidos que compartirán los profesores y una mayor in- 
teracción por parte de nuestros estudiantes, dejando a un lado las preocupaciones por el uso de una herramienta de video conferencia.

Muchas universidades e instituciones han incorporado en sus currículos académicos o en sus procesos de contratación, la Gestión Básica de Información como las habilidades mínimas necesarias para el desarrollo de los procesos de enseñanza, pero en la actualidad, estos deben ser criterios mínimos y habilidades de parte de nuestros usuarios. La tendencia es y será demostrar las habilidades necesarias para gestionar herramientas como Zoom, Teams, Gotomeeting, Google Meets, Big Blue Bottom, entre otras. En el menor tiempo, las instituciones de educación superior deberán adoptar y asegurar que la GBV (gestión Básica de Videoconferencias), contenga los requisitos mínimos para contratar y dictar una clase. Desde este momento veremos muchas instituciones implementando estas asignaturas en sus currículos y con el tiempo logrando que el uso de este, sea inherente al proceso de formación y así brindar garantías para que la innovación pedagógica dentro del aula virtual sea el artífice del aprendizaje y las herramientas pasen a un segundo plano.

Un tutor, profesor o monitor con formación en habilidades de GBV será una pieza idónea y preparada para asumir retos académicos en la enseñanza, diferentes a los de es- tar frente a una cámara y dictar su clase. Aquellos que logren tener estas competencias, lograrán hacer de un simple encuentro remoto, un espacio para fomentar estrategias como democracia en el aula virtual, votaciones en tiempo real o encuestas de apreciación rápida epolls, conformación de pequeños grupos de trabajo, incentivar el trabajo en equipos, uso adecuado de etiquetas en ambientes remotos y/o virtuales, afianzar seguridad en sus estudiantes. Con todo esto, seguramente sus procesos de enseñanza en la educación del futuro serán más transparentes y los beneficiados serán el cuerpo profesoral, la misma institución por tener personal idóneo para asumir los retos de la educación virtual y finalmente los estudiantes, por tener un espacio de aprendizaje adecuado, y por ofrecer las herramientas necesarias para que la innovación dentro de las herramientas de colaboración, brinde lo requerido para asegurar un aprendizaje con calidad.

\section{Laboratorio de innovación pedagógica: Una estrategia para el testing educativo - \\ innovación}

Para hablar de la educación del futuro, es imprescindible tener tecnología educativa de punta o tecnología con sentido de uso como apoyo a los procesos de formación, es por eso que nuestro factor (INNOVACIÓN) es uno de los pilares que no podrá faltar en el momento de hablar sobre educación virtual futura. 
En muchas instituciones, la innovación se contempla como la habilidad del profesor para hacer con poco, algo disruptivo, pero es necesario y demanda responsabilidad de las universidades y empresas para generar un espacio de Innovación Pedagógica Educativa; es así como aparece nuestra propuesta de Laboratorio de Innovación Pedagógica, como un espacio donde la sumatoria de tecnologías de punta y las necesidades de formación, deben converger para lograr experiencias disruptivas, innovadoras y estratégicas para los procesos de aprendizaje.

Un LIP tiene la particularidad que no solo brinda la creación de estas estrategias innovadoras, sino que también podrá ser el entorno necesario para lograr escritos, publicaciones, lineamientos y documentos necesarias para asegurar que la educación del futuro tenga calidad y orden en sus procesos.

Adicionalmente, un Laboratorio de Innovación Pedagógica permitirá a las instituciones tener proveedores aliados en tecnología de punta, creando socios estratégicos que le posibilitarán a las instituciones, realizar procesos de testing educativo para lograr suplir sus necesidades. Es claro que el núcleo de las tecnologías en los últimos años siempre es muy similar, Inteligencia Artificial, Machine Learning, Analítica Predictiva, entre otras; pero, si pensamos a futuro en nuestra educación, las empresas deberán de ser capaces de propiciar estos espacios para que desde las necesidades de formación se creen usos disruptivos e innovadores que generen aprendizaje en los usuarios finales.

Estos espacios deberán estar establecidos en todas las instituciones conformando un grupo interdisciplinar donde converjan áreas como el Departamento de Tecnología, profesores, proveedores, publicaciones, contratación, gestión humana, seguridad, jurídica, entre otros; todos estos, liderados por el centro de educación virtual y bajo este esquema, con seguridad las empresas tendrán sus lineamientos claros, sus tecnologías vigentes y con un alto uso, sus procesos de investigación activos. Y finalmente, una adopción correcta de las herramientas tecnológicas frente a las necesidades de aprendizaje y enseñanza de parte de sus usuarios. La educación del futuro deberá en todo momento tener y fomentar estos espacios en sus presupuestos y de manera acertada se consolidará como líder, pionero y estará preparada para afrontar cambios en la educación virtual como lo estamos viviendo hoy día.

\section{Datos no solo para informar, sino para renovar - analítica} Hemos llegado a nuestro último factor (ANALITICA). Esta es totalmente esencial cuando de educación presente y futura se trata, pero ¿cómo podemos proyectar la analítica en la educación virtual en un 
futuro? La respuesta la tenemos en la renovación de nuestros programas virtuales.

Tras el acuerdo 02 de 2020 donde el Consejo Nacional de Educación Superior (CESU). en conjunto con el Consejo Nacional de Acreditación (CNA), por el cual se actualiza el modelo de acreditación de alta calidad para instituciones y programas de educación superior en Colombia, la analítica ocupará un papel esencial en los procesos de acreditación o renovación de la oferta académica virtual. Con esto, el uso que se le brinde a este cúmulo de datos no solo tendrá como finalidad generar más información o tomas de decisiones, sino que será la fuente de información para que nuestros programas virtuales perduren en el tiempo.

La analítica resultante de los usos, la frecuencia, la pertinencia, el impacto y los accesos de los programas, aplicativos, plataformas y tecnologías educativas, en un futuro, será la evidencia tangible del impacto de la incorporación de las tecnologías y la eficacia en los procesos de enseñanza y aprendizaje obtenidos por las instituciones de educación superior. Debemos cambiar el "chip" y entender que la analítica no solo será para informar, sino para renovar.

Instituciones como la Universidad Northeastern está trabajando en poder identificar el uso y el impacto de las analíticas en la educación fu- tura, por lo cual cabe mencionar que, además de las posibilidades de tener datos capaces de facilitar la renovación de nuestros programas, también podremos impactar la educación en el futuro a través de:

- Identificar el ritmo y las necesidades de formación de nuestros estudiantes, es decir, al proyectar el proceso de enseñanza podremos decidir qué contenidos adaptativos brindaremos a nuestros usuarios, contenidos ajustados a la velocidad de la adquisición del saber y contenidos flexibles ajustados a la capacidad en que esta información sea digerida.

- Conocer la efectividad de la impartición de conocimiento por parte de la planta profesoral de una institución. Con esta información, la pertinencia de la información que se brinda desde la relación profesor-estudiante estará cuantificada y medida en razón a la calidad y el impacto de ésta en nuestros estudiantes.

- La toma de decisiones sobre el impacto de nuestros proyectos educativos basados en TIC en relación con la alineación de las estrategias educativas futuras y la visión empresarial.

La analítica será uno de los factores más decisivos en la educación virtual en el futuro y el punto de partida para renovar nuestras estrategias educativas basadas en tecno- 
logía. El fin último es lograr alinear los objetivos institucionales como también la forma en que dispondremos nuestros contenidos para asegurar una educación con calidad y un proceso de aprendizaje más claro para nuestros clientes, los estudiantes.

\section{Referencias}

Mylearningspace. (2014). Moodle and Cloud Computing.

Recuperado de

https://www.mylearningspace.com. au/news/moodle-and-cloud-compu ting

Valamis. (2021). Learning Experience Platform: Everything you need to know about LXP/LEP.

Recuperado de

https://www.valamis.com/hub/learn ing-experience-platform

Techradar.pro It Insights For Business. (2021). Best video conferencing software in 2021.

Recuperado de https://www.techradar.com/best/be st-video-conferencing-software

Kelsey Miller, University Northeastern. (2020). What is learning analytics \& how can it be used?

Recuperado de

https://www.northeastern.edu/

Ministerio de Educación Colombia. (2020). Acuerdo 02 del 1 de julio de 2020. Recuperado de https://www.mineducacion.gov.co/ 1759/w3-article-399567.html?_no redirect $=1$

Eric Spear, Precision Campus. (2020). 5 Big Benefits of Big Data Analytics in Education. Recuperado de https://precisioncampus.com/blog/ benefits-big-data-education/

Graspway LMS. (2020). ¿Qué es una Learning Experience Platform, LXP? Recuperado de https://www.graspway.com/que-esuna-learning-experience-platformIxp/

Gina Narcisi, Connecting the Australian Channel. (2020). 5 cool videoconferencing tips to make meetings more fun and effective.

Recuperado de

https://www.crn.com.au/news/5cool-videoconferencing-tips-to-ma ke-meetings-more-fun-and-effec tive-547434

Smartmind. (2020). Características y funciones de las Learning Experience Platform. LXP, la evolución de las plataformas e-learning.

Recuperado de

https://www.smartmind.net/blog/le arning-experience-platform-Ixpcaracteristicas-funciones/

Bryan Lovgren, Entrepreneur. (2020). Working Remote? These Are the Biggest Dos and Don'ts of Video Conferencing: As more and more businesses go remote; these are ways to be more effective and efficient on conference calls.

Recuperado de

https://www.entrepreneur.com/artic le/238902

Universidad del Rosario. (2020). Pilares de transformacion pregrado.

Recuperado de

https://www.urosario.edu.co/Pilare s-de-transformacion-pregrado/ini cio/ 
Co-Lab. (2019). Laboratorio de Innovación Educativa para la Educación Superior Co-LAB del Ministerio de Educación Nacional. Recuperado de https://colab.colombiaaprende.edu .col

Stanford University IT. (2019). Best Practices for Effective Video Conferencing.
Recuperado de https://uit.stanford.edu/videoconfer encing/best-practices.

Jhon Francined Herrera-Cubides, Nancy Yaneth Gelvez-García, Danilo Alfonso López-Sarmiento. (2018). LMS SaaS: Una alternativa para la formación virtual. Ingeniera. Revista chilena de ingeniería, vol. $27 \mathrm{~N}^{\circ} 1,2019$, pp. 164-179

\section{Manuel Antonio Meléndez Andrade.}

Ingeniero de Sistemas y Telecomunicaciones, amante del buen uso de las tecnologías educativas en las IES, ponente y consultor Moodle. Actualmente se desempeña como Profesional de Proyectos TIC para la Dirección de Educación Digital de la Universidad del Rosario Colombia y Director Académico del Segundo Encuentro para la Educación mediada por Tecnologías Digitales Reduc@te ACIS Colombia 2021. 